\title{
Screening in orbital-density-dependent functionals
}

\author{
Nicola Colonna, ${ }^{1}$ Ngoc Linh Nguyen, ${ }^{1}$ Andrea Ferretti, ${ }^{2}$ and Nicola Marzari ${ }^{1}$ \\ ${ }^{1}$ Theory and Simulation of Materials (THEOS) \\ and National Centre for Computational Design and Discovery of Novel Materials (MARVEL), \\ École Polytechnique Fédérale de Lausanne, 1015 Lausanne, Switzerland \\ ${ }^{2}$ Centro S3, CNR-Istituto Nanoscienze, 41125 Modena, Italy
}

(Dated: November 15, 2017)

\begin{abstract}
Electronic-structure functionals that include screening effects, such as Hubbard or Koopmans' functionals, require to describe the response of a system to the fractional addition or removal of an electron from an orbital or a manifold. Here, we present a general method to incorporate screening based on linear-response theory, and we apply it to the case of the orbital-by-orbital screening of Koopmans' functionals. We illustrate the importance of such generalization when dealing with challenging systems containing orbitals with very different chemical character, also highlighting the simple dependence of the screening on the localization of the orbitals. We choose a set of 46 transition-metal complexes for which experimental data and accurate many-body perturbation theory calculations are available. When compared to experiment, results for ionization potentials show a very good performance with a mean absolute error of $0.2 \mathrm{eV}$, comparable to the most accurate many-body perturbation theory approaches. These results reiterate the role of Koopmans' compliant functionals as simple and accurate quasiparticle approximations to the exact spectral functional, bypassing diagrammatic expansions and relying only on the physics of the local density or generalized-gradient approximation.
\end{abstract}

\section{INTRODUCTION}

Accurate prediction of ground- and excited state properties of molecules can be made using quantum chemistry wave-function method or many-body perturbation theory (MBPT) techniques ${ }^{1}$. However such calculations scale unfavorably with the size of the system and become soon computationally untreatable. For this reason electronic-structure approaches such as Hartree Fock (HF) or Kohn-Sham density-functional theory (KS-DFT) have often been used as a proxy to classify and understand excitation spectra. However, the eigenvalues of the KS potential have no obvious relationship with the real excited states of the system. One notable exception is the first ionization potential in finite systems, that is exactly reproduced in exact KS-DFT ${ }^{2}$, , but usually severely underestimated by standard local or semilocal approximations to the exchange-correlation energy functional. In HF theory, the single-particle energies do have the physical meaning of excitation energies thanks to Koopmans' theorem, but miss important relaxation effects related to the addition of an electron (or a hole) to the system. These effects can be included in finite systems using e.g. $\Delta$ self consistent field $(\Delta \mathrm{SCF})$ calculations ${ }^{1}$, where the change in the energy associated with an electron addition/removal is calculated via two self-consistent calculations done with $\mathrm{N}$ and $\mathrm{N}+1 / \mathrm{N}-1$ electrons. However, it is not straightforward to apply this approach to singleparticle energies beyond the frontier ones and its extension to solids poses some issues since the $\triangle \mathrm{SCF}$ correction, computed with standard density functional approximations, vanishes in the thermodynamic limit.677

For these reasons it would be highly desirable to have a functional yielding accurate single particle energies in addition to the well-established accuracy for ground state properties. Failures of standard density functional approximations in reproducing spectral quantities, such as ionization potentials and electron affinities, has been connected to the deviation from piecewise linearity (PWL) of the the total energy functional as a function of particle number, and the associated lack of derivative discontinuity at integer particle numbers. First, the deviation from PWL has been suggested $\frac{812}{12}$ as a definition of electronic self-interaction errors $\frac{13}{13}$, and in recently developed functionals, such as DFT-corrected 14 17 , rangeseparated $\frac{18}{20}$ or dielectric-dependent hybrid functionals 21 23, PWL has been recognized as a critical feature to address. The criterion of PWL was in particular chosen as a key feature by some of us to introduce the class of Koopmans-compliant (KC) functionals 24 28. When used to purify approximate standard local or semilocal density functionals, Koopmans' corrections lead to orbitaldensity dependent potentials that can be interpreted $\frac{29}{29}$ an approximation of the spectral potential, i.e. the local and dynamical potential that is necessary and sufficient to describe the local spectral density and, in turn, photoemission spectra ${ }^{30}$. With a relatively small increase of the computational cost this class of functionals delivers accurate spectral properties retaining (in some cases exactly) the good performance of the underlying DFT functional for the ground state total energy 28 . The performance of the $\mathrm{KC}$ predictions greatly depends on the correct description of screening/relaxation effects associated to particle number modifications. For simple systems, these effects can be effectively captured by introducing a screening coefficient derived imposing the generalized Koopmans theorem on the frontier orbita ${ }^{25 \mid 28}$. However, systems with a diverse electronic manifold, like for instance transition-metal compounds, call for a more accurate treatment since the same coefficient cannot equally 
well describe screening when the electron is removed (or added) e.g. from an $s$ - or a $d$-like orbital. In this work we discuss how to correctly and fully include screening via orbital-dependent coefficients obtained from a linear response theory and thus ultimately from the dielectric screening. We then analyze the performance of the Koopmans-Integral (KI) functional28, a standard flavour of the $\mathrm{KC}$ class whose definition will be reviewed in Sec. II] for a set of 46 transition-metal complexes, and validate the results against $\mathrm{GW}$ calculations and experiments.

The paper is organized as follow. In section II] we review the basic feature of Koopmans-compliant functionals and we introduce the linear-response approach to screening and relaxation. In section III we compare ionization potentials for the set of molecules under study, obtained at different level of theory, with experimental data and state-of-the-art many-body perturbation theory calculations, followed by summary and conclusions.

\section{METHOD AND COMPUTATIONAL DETAILS}

In this section the main features of the $\mathrm{KC}$ class of functionals are reviewed with particular emphasis on the
KI flavour, and the new scheme to capture screening and relaxation effects based on a linear-response approach is introduced and discussed.

\section{A. Koopmans-compliant functionals}

Koopmans-compliant functionals 24,28 explicitly enforce a generalized criterion of piecewise linearity (PWL) with respect to the fractional removal/addition of an electron from any orbital in approximate DFT functionals. This is done by removing orbital-by-orbital the Slater contribution to the total energy, an approximately quadratic term in the orbital occupation $f_{i}$, and replacing it with a linear (Koopmans) term. In practice, the Koopmans correction is made up by the product of two equally important terms: $(i)$ an orbital-density dependent corrections $\Pi_{i}^{\mathrm{u}}$ aiming at correctly describing the addition/removal of an electron in a frozenorbital or unrelaxed picture (restricted Koopmans theorem), and ( $i i)$ a screening factor $\alpha_{i}$ which takes into account the relaxation of the orbitals as a response to the addition/removal process. In what follows we restrict our attention to the KI energy functiona 28 , defined as $E^{\mathrm{KI}}=E^{\mathrm{DFT}}+\sum_{i} \alpha_{i} \Pi_{i}^{\mathrm{uKI}}$, where

$$
\begin{aligned}
\Pi_{i}^{\mathrm{uKI}}\left(f_{i}\right) & =-\int_{0}^{f_{i}} d s\left\langle\psi_{i}\left|H^{\mathrm{DFT}}(s)\right| \psi_{i}\right\rangle+f_{i} \int_{0}^{1} d s\left\langle\psi_{i}\left|H^{\mathrm{DFT}}(s)\right| \psi_{i}\right\rangle \\
& =-\left\{E^{\mathrm{DFT}}\left[\rho_{u}\right]-E^{\mathrm{DFT}}\left[\rho_{u}^{f_{i}=0}\right]\right\}+f_{i}\left\{E^{\mathrm{DFT}}\left[\rho_{u}^{f_{i}=1}\right]-E^{\mathrm{DFT}}\left[\rho_{u}^{f_{i}=0}\right]\right\}
\end{aligned}
$$

is the unrelaxed KI correction to the DFT energy (see Ref. 28 for a detailed discussion of the KI energy functional and KI orbital-dependent potentials) and $\alpha_{i}$ the orbital dependent screening factor. Here the orbitals $\left\{\psi_{i}\right\}$ are kept frozen, $\rho_{u}=\sum_{j} f_{j}\left|\psi_{j}\right|^{2}$ is the total density of the system and $H^{\mathrm{DFT}}(s)$ is the approximate KSDFT Hamiltonian calculated at the unrelaxed density $\rho_{u}^{f_{i}=s}=\sum_{j \neq i} f_{j}\left|\psi_{j}\right|^{2}+s\left|\psi_{i}\right|^{2}$, where only the explicit dependence on the occupation $s$ is considered [the $f_{j}$ for $j \neq i$ are typically 1 (or 2 for spin degeneracy)].

At variance with DFT, the variation of the Koopmanscompliant functionals (and ODD functionals in general) leads to local but orbital-dependent Hamiltonians that are in general not invariant under unitary transformations of the electronic wavefunctions. The energy minimization defines a unique set of variational orbitals that are usually very localized $\frac{28 \mid 31+35}{35}$ in space and resemble Boys orbitals 36 or maximally localized Wannier functions 37 . At the minimum, the matrix of Lagrangian multipliers $\Lambda$, associated to the orthogonality constraint, becomes Hermitian 38 and can be diagonalized via a unitary transformation, allowing $\sqrt{32 / 41}$ one to define a second set of canonical orbitals, usually less localized than the variational ones. Although still a debated point ${ }^{42}$, it is a common practice to interpret the eigenvalues of $\Lambda$ as (canonical) orbital energies32|41|43, as clearly argued for in Ref. 39 and 29 .

We note that the KI energy functional at integer occupation numbers preserves the unitary invariance of the underlying DFT functional28. The energy minimization is therefore not sufficient to uniquely determine the variational orbitals; at the same time the KI potentials defining the KI eigenvalues depend on the actual representation of the electronic manifold 28 . In previous works ${ }^{28|44| 45}$ we removed this ambiguity by adding to the KI energy functional a vanishingly small Perdew-Zunger (PZ) self-interaction correction, thus interpreting KI as the limit of the KIPZ functional ${ }^{28}$ when the PZ correction goes to zero. The small PZ correction breaks the unitary invariance, and leads, through the energy functional minimization, to a set of well defined and typically localized variational orbitals (PZs orbitals in the following) on which the KI corrections are computed. This choice is of course arbitrary, although having a localized set of the 
orbitals is a key enabler for the good performance of $\mathrm{KC}$ functionals, and a mandatory one when extended systems are considered $\frac{46}{6}$. This is confirmed also here, by applying the KI corrections on different sets of orbitals with different degrees of localization; beside the set defined by the limiting procedure described above (PZs) we also consider the one represented by the canonical (KS) orbitals of the base DFT functional (usually delocalized over the whole molecule) and other two localized representations given by maximally localized Wannier functions (MLWFs) and by atomic-projected Wannier functions (ProjWFs). The latter are obtained by a projection of the KS states on a set of atomic orbitals that serves as a rough guess for the Wannier function, followed by a symmetric orthonormalization. For the MLWFs, the localization is enforced by using the sum of the quadratic spreads of the wavefunctions as a localization criterion, and searching for the optimal unitary transformation that satisfies that criterion. ${ }^{37}$ The use of Wannier functions as a representation to apply the Koopmans' corrections and restore the missing piecewise linearity of approximate DFT functionals has been shown to give good results for the band gaps of solids 4 . In the following we use the notation $\mathrm{KI} @$ [representation] to indicate the set of orbitals used to compute the KI corrections.

The screening and relaxation effects, naturally associated with the removal/addition of an electron, are accounted for by the multiplicative factor $\alpha_{i}$ in front of the bare correction $\Pi_{i}^{\mathrm{uKI}}$. How to determine this screening is the central goal of this work. In previous applications we showed that a unique (identical for all valence orbitals) screening factor, chosen to enforce the generalized Koopmans' condition on the frontier orbital ${ }^{25 \mid 28}$, is sufficient to accurately predict the ionization potentials (IPs) and also photoemission spectra in a variety of molecular systems $28|44| 45$. A second screening coefficient, derived imposing the Koopmans' condition on the lowest unoccupied moleclar orbital (LUMO), can be attached to all the conduction states, extending the predictive power of $\mathrm{KC}$ functionals to electron affinities (EAs) ${ }^{44 / 45}$. However, a more sophisticated orbital-dependent choice is needed in case of systems with a more diverse electronic manifold. In the next section we present and discuss this more general, physically sound and orbital-dependent treatment of the screening based on linear-response theory 4

\section{B. Screening in $\mathrm{KC}$ functionals}

In order to revert the unrelaxed Koopmans' correction to a fully relaxed one, the screening coefficients can be formally defined as $\alpha_{i}=\Pi_{i}^{\mathrm{rKI}} / \Pi_{i}^{\mathrm{uKI}}$ where $\Pi_{i}^{\mathrm{rKI}}$ is the relaxed Koopmans correction defined in Ref. 25. The latter can be written as

$$
\Pi_{i}^{\mathrm{rKI}}\left(f_{i}\right)=-\left\{E^{\mathrm{DFT}}[\rho]-E^{\mathrm{DFT}}\left[\rho^{f_{i}=0}\right]\right\}+f_{i}\left\{E^{\mathrm{DFT}}\left[\rho^{f_{i}=1}\right]-E^{\mathrm{DFT}}\left[\rho^{f_{i}=0}\right]\right\}
$$

where, at variance with Eq. 11), $\rho^{f_{i}=s}$ in this expression corresponds to the fully relaxed density compatible with the condition $f_{i}=s$. Total energies with the additional constraint on one of the occupation numbers appearing in Eq. (2), could in principle be evaluated by means of constrained-density-functional approach 50 . Applications have been made to e.g. coulomb-interaction parameters to be used in model Hamiltoniant $51+53$ or in the context of Hubbard $U$ correction to DFT ${ }^{8}$. Here we follow an alternative route, and evaluate each term in Eq. (2) in a perturbative way introducing a Taylor expansion of the DFT energy with respect to the occupation $f_{i}$ around some reference occupation $f_{\text {ref }}$ :

$$
E^{\mathrm{DFT}}\left[\rho^{f_{i}=s}\right]=\left.\sum_{n} \frac{1}{n !} \frac{d^{n} E^{\mathrm{DFT}}}{d f_{i}^{n}}\right|_{f_{r e f}}\left(s-f_{\mathrm{ref}}\right)^{n} .
$$

Substituting in Eq. (2) and stopping at the second order we find:

$$
\Pi_{i}^{\mathrm{rKI}}\left(f_{i}\right)=\left.\frac{1}{2} f_{i}\left(1-f_{i}\right) \frac{d^{2} E^{\mathrm{DFT}}}{d f_{i}^{2}}\right|_{f_{\mathrm{ref}}}+O\left(f_{i}^{3}\right) .
$$

Due to Janak's theorem ${ }^{54}$ the second derivative of the energy wrt a given occupation represents also the first derivative of the corresponding eigenvalue. In the following, we will work in the diagonal representation of the KS-DFT Hamiltonian ${ }^{55}$ and in the general case of relaxed orbitals, the frozen-orbital case being recovered trivially at the end of the derivation. We have:

$$
\begin{aligned}
\frac{d^{2} E^{\mathrm{DFT}}}{d f_{i}^{2}} & =\frac{d \varepsilon_{i}}{d f_{i}}=\left\langle\psi_{i}\left|\frac{d v_{\mathrm{Hxc}}}{d f_{i}}\right| \psi_{i}\right\rangle \\
& =\int d \mathbf{r} d \mathbf{r}^{\prime} n_{i}(\mathbf{r}) f_{\mathrm{Hxc}}[\rho]\left(\mathbf{r}, \mathbf{r}^{\prime}\right) \frac{d \rho\left(\mathbf{r}^{\prime}\right)}{d f_{i}}
\end{aligned}
$$

where we have used the Hellmann-Feynman theorem $\underline{56157}$ in the second identity, and have introduced the orbital density $n_{i}=\left|\psi_{i}\right|^{2}$ and the Hartree-exchange-correlation (Hxc) kernel $f_{\mathrm{Hxc}}=\delta^{2} E_{\mathrm{Hxc}} / \delta \rho^{258159}$. The derivative of the charge density with respect to the occupation is made up by two contributions: the first one comes from the explicit dependence of the density on the occupations while the second one comes from the change in the orbitals at 
fixed occupation, i.e. at fixed number of particles:

$$
\begin{aligned}
\frac{d \rho(\mathbf{r})}{d f_{i}} & =n_{i}(\mathbf{r})+\int d \mathbf{r}^{\prime} \frac{\delta \rho(\mathbf{r})}{\delta v_{\mathrm{KS}}\left(\mathbf{r}^{\prime}\right)} \frac{d v_{\mathrm{KS}}\left(\mathbf{r}^{\prime}\right)}{d f_{i}} \\
& =n_{i}(\mathbf{r})+\int d \mathbf{r}^{\prime} \chi_{0}\left(\mathbf{r}, \mathbf{r}^{\prime}\right) \frac{d v_{\mathrm{Hxc}}\left(\mathbf{r}^{\prime}\right)}{d f_{i}} \\
& =n_{i}(\mathbf{r})+\int d \mathbf{r}^{\prime}\left[\chi_{0} f_{\mathrm{Hxc}}\right]_{\left(\mathbf{r}, \mathbf{r}^{\prime}\right)} \frac{d \rho\left(\mathbf{r}^{\prime}\right)}{d f_{i}}
\end{aligned}
$$

where $\chi_{0}=\delta \rho / \delta v_{\mathrm{KS}}$ is the KS (non-interacting) densitydensity response function. Equation (6) is a Dyson-like equation for the derivative of the charge density. Its iterative solution can be recast in a compact form introducing the interacting density-density response function $\chi=\chi_{0}+\chi_{0} f_{\mathrm{Hxc}} \chi^{49158}$.

$$
\frac{d \rho(\mathbf{r})}{d f_{i}}=n_{i}(\mathbf{r})+\int d \mathbf{r}^{\prime}\left[\chi f_{\mathrm{Hxc}}\right]_{\left(\mathbf{r}, \mathbf{r}^{\prime}\right)} n_{i}\left(\mathbf{r}^{\prime}\right) .
$$

where it is understood that the response function and the Hxc-kernel are evaluated at $\rho=\rho^{f_{i}=f_{\text {ref }}}$. In the frozen orbital approximation the second term on the right hand side of the equation above is exactly zero. Combining Eqs. (7), (5) and (4) we obtain the central result of this paper:

$$
\begin{aligned}
& \Pi_{i}^{(2) \mathrm{uKI}}\left(f_{i}\right)=\frac{1}{2} f_{i}\left(1-f_{i}\right) \int d \mathbf{r} d \mathbf{r}^{\prime} n_{i}(\mathbf{r}) f_{\mathrm{Hxc}}\left(\mathbf{r}, \mathbf{r}^{\prime}\right) n_{i}\left(\mathbf{r}^{\prime}\right) \\
& \Pi_{i}^{(2) \mathrm{rKI}}\left(f_{i}\right)=\frac{1}{2} f_{i}\left(1-f_{i}\right) \int d \mathbf{r} d \mathbf{r}^{\prime} n_{i}(\mathbf{r}) \mathcal{F}_{\mathrm{Hxc}}\left(\mathbf{r}, \mathbf{r}^{\prime}\right) n_{i}\left(\mathbf{r}^{\prime}\right)
\end{aligned}
$$

where we have defined the screened Hxc-kernel $\mathcal{F}_{\mathrm{Hxc}}=$ $\left(I+f_{\mathrm{Hxc}} \chi\right) f_{\mathrm{Hxc}}=\epsilon^{-1} f_{\mathrm{Hxc}}$. It is important to stress that all the quantities needed to evaluate Eqs. (8), i.e. the orbital density $n_{i}(\mathbf{r})$, the Hxc kernel $f_{\mathrm{Hxc}}$ and the response functions $\chi_{0}$ and $\chi$, are all ground-state properties and, therefore, accessible from the reference ground state calculation (usually the one for the neutral system). Instead, in a finite difference approach, as the one adopted in Refs. 46 47 to compute energy differences when changing the occupation numbers, one needs to ask for the additional requirement to keep fixed the orbital where the electron is added or removed. This is to prevent it from morphing into the highest (partially) occupied orbital, as this would always be the most favorable energetic configuration because of the Aufbau principle. In the linear response approach described above this is not needed since one always refers to single-particle orbitals and energies of the reference calculation, which are fixed by construction.

Even if derived from a simplified treatment based on a second order Taylor expansion, it is formally evident that the inclusion of orbital relaxation leads to a screening of the unrelaxed Koopmans correction. The integrals appearing in Eq. (8) can be interpreted as the effective interaction between the electrons in the orbital $i$ when all the other orbitals are allowed (second line) or not (first line) to readjust. The connection between the second derivative of the energy with respect to the occupation of a localized orbital and the effective interaction between localized electrons has been also discussed in the context of Hubbard corrections to DFT 60 62 The value of the static $U$ parameter to be used in the model Hamiltonian is indeed determined from the constrained variation of the DFT eigenvalue with respect to the occupation number of the localized orbitals 8151 53/63/64. The frequencydependence of the effective interaction has been also computed within the constrained random-phase approximation $\underline{65}$ (cRPA) using an expression similar to the second line of Eq. (8) but evaluated at the RPA level, i.e. neglecting the xc-kernel both in the Dyson-like equation defining the response function $\chi$ and in the dielectric matrix $\epsilon$. Within this second order expansion the similarity of the Koopmans-compliant functional with the $+U$ correction in DFT is even more evident and indeed these functionals can be interpreted as a generalization of the DFT $+\mathrm{U}$ approach to the entire electronic manifold (this was the reason, in primis, of their introduction"2425). Notwithstanding the apparent similarity, there is a fundamental difference between the two approaches in the fact that $\mathrm{KC}$ corrections to DFT aim at describing addition or removal of an electron from the system (charged excitation), while the $+U$ correction can be interpreted as the energy cost associated to move a fraction of an electron from a localized orbital (or manifold) to the bath represented by the rest of the system (neutral excitation). Then, as argued in Ref. 69, the renormalization of the bare interaction might take place through different screening channels depending on whether the electron is added/removed or continues to stay in the system. In the derivation above, the explicit variation of the particle number is considered [first contribution in Eq. [7]], as KC functionals aim at describing charged excitations, and the screened Koopmans' correction in Eq. (8) is thus the correct one for such processes. Most importantly, the central results of Eq. (8) are still valid if one substitutes the canonical set of KS orbitals with another set related to the first one by a unitary transformation (see Supporting Information); this is extremely important because the screening coefficients to be used in $\mathrm{KC}$ functionals are actually connected to the variational orbitals, i.e. the orbitals that minimize the $\mathrm{KC}$ functionals, and these are related by a unitary transformation to the canonical orbitals.

The second line in Eq. (8) could be used as a relaxed Koopmans correction, albeit exact only up to second order. We therefore continue to use the original definition of the screening, and we introduce an orbital-dependent screening coefficient $\alpha_{i}$ defined as the ratio between the relaxed and unrelaxed second-order Koopmans correction:

$$
\alpha_{i}=\frac{\Pi_{i}^{(2) \mathrm{rKI}}\left(f_{i}\right)}{\Pi_{i}^{(2) \mathrm{uKI}}\left(f_{i}\right)}=\frac{\left\langle n_{i}\left|\mathcal{F}_{\mathrm{Hxc}}\right| n_{i}\right\rangle}{\left\langle n_{i}\left|f_{\mathrm{Hxc}}\right| n_{i}\right\rangle}
$$

with $\left\langle n_{i}|A| n_{i}\right\rangle=\int d \mathbf{r} d \mathbf{r}^{\prime} n_{i}(\mathbf{r}) A\left(\mathbf{r}, \mathbf{r}^{\prime}\right) n_{i}\left(\mathbf{r}^{\prime}\right)$. The simplified screening coefficient introduced in previous pub- 
lications ${ }^{25 \mid 28}$ can be seen as a particular case of the present approach reducing the electronic screening function $\epsilon^{-1}\left(\mathbf{r}, \mathbf{r}^{\prime}\right)$ to a constant. We also note that the definition of the screening coefficient in Eq. (9) is similar to the expression of the mixing parameter in the context of dielectric-dependent hybrid functionals ${ }^{70}$ generalized to finite systems 23171 .

Screening coefficients have been efficiently computed resorting to the linear-response approach of densityfunctional perturbation theory (DFPT) ${ }^{73}$. The second term in the right hand side of Eq. (7) can be indeed interpreted as the density variation due to a "bare" perturbing potential $\Delta^{i} V(\mathbf{r})=\Delta f_{i} \int d \mathbf{r}^{\prime} f_{\mathrm{Hxc}}\left(\mathbf{r}, \mathbf{r}^{\prime}\right) n_{i}\left(\mathbf{r}^{\prime}\right)$. This density variation is equivalent to that induced in the auxiliary KS system by an effective potential $\Delta^{i} V_{\text {eff }}(\mathbf{r})=$ $\Delta^{i} V(\mathbf{r})+\Delta^{i} V_{\mathrm{Hxc}}(\mathbf{r})$ with $\Delta^{i} V_{\mathrm{Hxc}}$ being the self consistent variation of the Hxc potential due to the change in the density. The linear-response calculation of the screening coefficient has been implemented in a modified version of the Phonon code of QUANTUM ESPRESSO ${ }^{74 / 75}$.

\section{Computational setup}

We apply this complete orbital-dependent scheme to compute the IPs of a set of 46 transition metal complexes first introduced in Ref. 72, for which experimental as well as many body perturbation theory results are available (only 41 values are available from experiments). The calculations are performed using Optimized NormConserving Vanderbilt (ONCV) pseudopotentials ${ }^{76}$ to model the interaction between nuclei and electrons. In order to simulate isolated molecules ${ }^{79}$ we place these inside an orthorombic cell with 22 Bohr of vacuum in each direction, sufficient to converge total energies and single particle eigenvalues when the Coulomb interaction between periodic images are suppressed using reciprocalspace counter-charge corrections 80 . The energy cut-off for the plane-wave expansion is set to $100 \mathrm{Ry}$ (400 Ry for the charge density). All orbital-density dependent calculations presented here uses $\mathrm{PBE}^{81}$ as the underlying xc-energy functional; energy minimizations are performed on the space of complex wavefunctions.

The calculated IP is defined as minus the eigenvalue of the highest-occupied molecular orbital (HOMO) IP = $-\varepsilon_{h o}$ for all the theoretical methods reported, except for $\triangle \mathrm{SCF}$ when the IP is defined as the energy difference between the neutral molecule and its cation.

\section{RESULTS AND DISCUSSION}

All the calculated and available experimental IPs are listed in the Supporting Information. We focus below on the average performance of the KI functional as estimated by the mean absolute error (MAE), mean signed error (MSE) and maximum signed error $[\operatorname{Max}(+)$, $\operatorname{Max}(-)]$.

\section{A. Performance of the KI functional}

In Fig. 1 the absolute difference between calculated and experimental ionization energies (IPs) is drawn as a color map. In Tab. I the resulting mean absolute error (MAE), mean signed error (MSE) and maximum signed error $[\operatorname{Max}(+), \operatorname{Max}(-)]$ are listed 82 . The PBE results show the well-known underestimation of the IPs due to the self-interaction error resulting in a MAE and a maximum signed error of 3.43 and $-5.27 \mathrm{eV}$, respectively. The self-interaction correction (SIC) by Perdew and Zunger 13 (PZ-SIC in the Figures and Tables) over-corrects the PBE results with an almost systematic overestimation of the IP, while $\triangle \mathrm{SCF}$, that usually gives rather good estimation of the frontier orbitals energies, in this case has a mean absolute error of $0.65 \mathrm{eV}$ which is only slightly smaller than PZ-SIC. KI corrections computed on top of the canonical PBE orbitals (KI@KS) show an average performance that is comparable to that of $\triangle \mathrm{SCF}$. The two methods perform very similarly for each single molecule (see Fig. 2 and Tab. I in Supporting Information); this is not by chance, and highlights the physical content embedded in the KI corrections: When relaxation effects are correctly taken into account the KI functional reverts the $\mathrm{KS}$ eigenvalues into a $\triangle \mathrm{SCF}$ energy, inheriting the accuracy of finite-difference DFT energies. The use of a localized representation leads to a significant improvement of the performance with a reduction of the MAE by a factor $2 \div 3$ over $\triangle \mathrm{SCF}$ and KI@KS. The KI@ProjWF and the KI@PZs show very similar average performance with a MAE comparable to those from the best $\mathrm{GW}$ calculations, i.e. $\mathrm{G}_{0} \mathrm{~W}_{0} @ \mathrm{PBE} 0$ (MAE $0.21 \mathrm{eV}$ ), chosen among ten different prescriptions ${ }^{72}$. We stress here that all the theoretical IPs do not include relativistic nor zero-point motion effects. As highlighted in Refs. 72 83, the latter is usually negligible while the former might be more relevant for this set of molecules and affect the comparison between theory and experiment. Comparison between different theoretical methods is instead fully consistent since these all neglect relativistic effects and zero-point motion.

In order to highlight the importance of the orbitaldependent treatment of the screening we also show the KI@PZs results obtained using a unique value of the screening coefficient, i.e. $\alpha_{i}=\alpha_{c n} \quad \forall i$ (uniq. $\alpha$ in plots and Tables). Here the subscript " $c n$ " stands for "cation-neutral" and points to the fact that $\alpha_{c n}$ is calculated $^{25 \mid 28145}$ imposing the generalized Koopmans condition on the HOMO, i.e. requiring that the HOMO eigenvalue of the neutral system is equal to the LUMO eigenvalue of the same system deprived by one electron: $\operatorname{IP}^{\mathrm{N}}\left(\alpha_{\mathrm{cn}}\right)=\mathrm{EA}^{(\mathrm{N}-1)}\left(\alpha_{\mathrm{cn}}\right)$. A comparison between $\mathrm{KI} @ \mathrm{PZs}$ with and without the orbital-dependent treatment of the screening reveals that the MAE and the maximum errors are almost halved highlighting the importance of a more advanced treatment of the screening for transition-metal complexes. We also stress here that the way of computing a unique screening coefficient, although 
FIG. 1. Absolute difference in eV between calculated and experimental ionization potentials for different level of theory. The $\mathrm{G}_{0} \mathrm{~W}_{0} @ \mathrm{PBE} 0$ results are from Ref. 72 (see Supporting information for a full table).

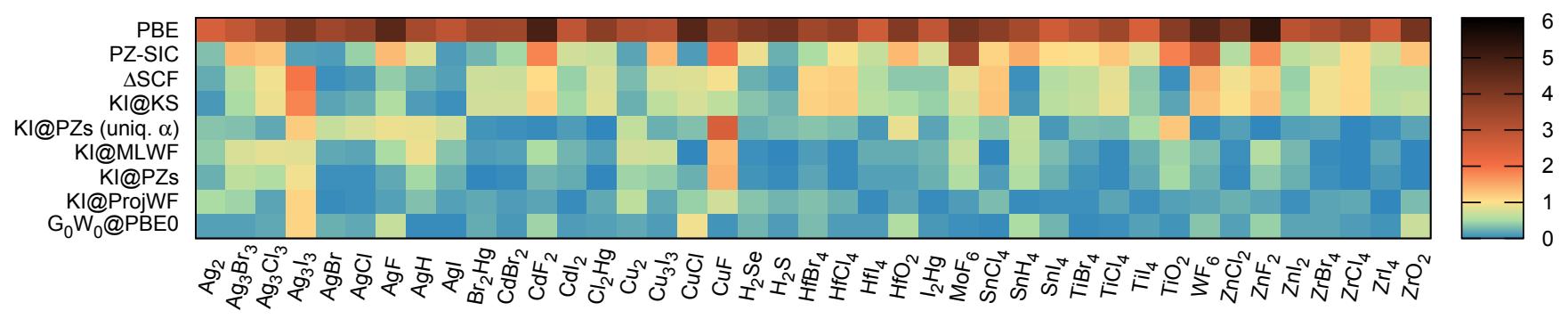

\begin{tabular}{|c|c|c|c|c|c|c|c|c|c|}
\hline & PBE & PZ-SIC & $\Delta \mathrm{SCF}$ & KI@KS & $\begin{array}{l}\text { KI@PZs } \\
\text { (uniq. } \alpha \text { ) }\end{array}$ & KI @MLWF & $\mathrm{KI} @ \mathrm{PZs}$ & KI @ProjWF & $\mathrm{G}_{0} \mathrm{~W}_{0} @ \mathrm{PBE} 0$ \\
\hline MAE & 3.43 & 0.87 & 0.62 & 0.63 & 0.39 & 0.29 & 0.22 & 0.20 & 0.21 \\
\hline MSE & -3.43 & 0.82 & -0.51 & -0.57 & 0.28 & 0.27 & 0.18 & -0.02 & -0.07 \\
\hline $\operatorname{Max}(+)$ & - & $\begin{array}{c}1.97 \\
(\mathrm{CuF})\end{array}$ & $\begin{array}{c}0.96 \\
(\mathrm{CuF})\end{array}$ & $\begin{array}{c}0.63 \\
(\mathrm{CuF})\end{array}$ & $\begin{array}{c}2.51 \\
(\mathrm{CuF})\end{array}$ & $\begin{array}{c}1.37 \\
(\mathrm{CuF})\end{array}$ & $\begin{array}{c}1.43 \\
(\mathrm{CuF})\end{array}$ & $\begin{array}{c}0.74 \\
(\mathrm{CuF})\end{array}$ & $\begin{array}{c}0.53 \\
\left(\mathrm{SnH}_{4}\right)\end{array}$ \\
\hline $\operatorname{Max}(-)$ & $\begin{array}{c}5.27 \\
\left(\mathrm{ZnF}_{2}\right)\end{array}$ & $\begin{array}{c}0.35 \\
\left(\mathrm{Ag}_{2}\right)\end{array}$ & $\begin{array}{c}1.25 \\
\left(\mathrm{SnCl}_{4}\right)\end{array}$ & $\begin{array}{c}1.30 \\
\left(\mathrm{ZnF}_{2}\right)\end{array}$ & $\begin{array}{c}0.37 \\
\left(\mathrm{HfBr}_{4}\right)\end{array}$ & $\begin{array}{c}0.12 \\
\left(\mathrm{HfBr}_{4}\right)\end{array}$ & $\begin{array}{c}0.19 \\
\left(\mathrm{HfBr}_{4}\right)\end{array}$ & $\begin{array}{c}0.37 \\
\left(\mathrm{H}_{2} \mathrm{Se}\right)\end{array}$ & $\begin{array}{c}0.47 \\
\left(\mathrm{CdF}_{2}\right)\end{array}$ \\
\hline
\end{tabular}

TABLE I. Mean absolute error (MAE), mean signed error (MSE) and maximum (positive and negative) deviation between calculated and experimental ionization potentials for different level of theory (see text). For PBE all the errors are negative and only the maximum negative error makes sense and is reported. The $\mathrm{G}_{0} \mathrm{~W}_{0} @ \mathrm{PBE} 0$ calculations are from Ref. 72 . All the data are in $\mathrm{eV}$. The system corresponding to the maximum error is also reported in parenthesis.

conceptually straightforward and appealing, is cumbersome to extend to orbitals different from the frontier ones, and also requires in practice multiple calculations at $\mathrm{N}$ and N-1 electrons 28 . The linear-response approach introduced here bypasses both these problems in a natural way.

For all our KI calculations, the outlier with the largest error with respect to experimental $\mathrm{IP}$ is the $\mathrm{CuF}$ molecule. It is highly unlikely that the large deviations observed for this molecule (see Tab. II) comes from effects not included in our calculations. Finite temperature and relativistic effects and/or zero-point motion could account for discrepancy of the order of few tenths of an electron volt ${ }^{72}$. However, it should be also mentioned that the experiment values usually come with an error bar and in this particular case the experimentally

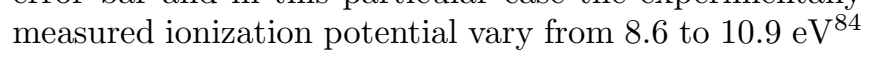
making any comparison not particularly significant.

\section{B. Effect of the localized representation}

It's interesting to note that despite some inevitable dependence on the choice of the localized representation, there is a substantial equivalence between the KI cal- culations done on top of the 3 different localized set of orbitals. In Fig. 2 we try to quantify such dependence of the IPs on the underlying representation defining the mean absolute distance (MAD) between two sets ( $a$ and $b$ ) of IPs from different KI calculations as

$$
\operatorname{MAD}_{a b}=\frac{1}{N} \sum_{i=1}^{N}\left|\mathrm{IP}_{i}^{a}-\mathrm{IP}_{i}^{b}\right|,
$$

and plotting it as a color map. We also add the $\triangle \mathrm{SCF}$ results to highlight again its close relation with KI@KS. The MAD between these two sets of IPs is only 0.08 $\mathrm{eV}$. From the correlation matrix one can also see that KI@PZs and KI@MLW are the second closest pair, reflecting the fact that the PZ localization condition and the MLW one usually lead to very similar sets of orbitals. We also clearly see that calculations on localized sets of orbitals and calculations on KS states (KI@KS and $\triangle \mathrm{SCF}$ ) form two distinct blocks with off-diagonal elements up to $1 \mathrm{eV}$. The better performance of KI when a localized representation is used with respect to the KI@KS emphasizes what is a key requirement for this class of functionals, i.e. expressing the Koopmans corrections on a localized set of orbitals $\sqrt{46}$. While the discrepancy is not dramatic in the case of atoms or small 


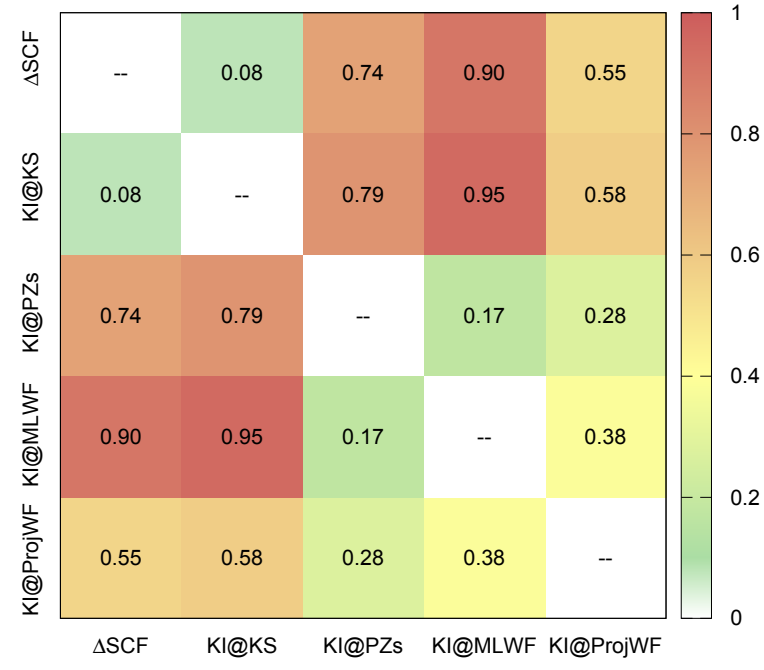

FIG. 2. Mean absolute distance (see text) between the results of the different KI calculations.

molecules, it becomes more and more evident when increasing the size of the system; given the strong connection with the $\triangle \mathrm{SCF}$ method, KI@KS would experience the same failure of the finite-difference method in the thermodynamic limit 617 . Using a localized representation of the electronic manifold to compute the orbital corrections ensures instead a finite correction also in the thermodynamic limit $\frac{46}{6}$. In addition, there is a strong correlation between screening parameters and orbital localization, e.g. as described by the self-Hartree energies (see Supporting Information). Such correlations would make it easier to apply this approach to large scale calculations, where screening coefficients could be inferred from a few linear-response tests.

Before concluding, we mention two points that are also relevant to the present discussion and worthy of future studies. First, system symmetries are not necessarily reproduced by orbital-density dependent functionals 39185 , if the corresponding Hamiltonians do not preserve these - hence the symmetry of the localized representation can play an important role, especially in small systems or in the atomic limit. Second, the present discussion makes clear, as is already known for the case of Hubbard functionals 86187 , that derivatives of the total energy would need to take into account the dependence of the screening coefficients on the varying parameters, e.g. atomic displacements. This dependence is expected to be small or negligible, and in any case these functionals are meant to great enhance the spectral properties, leaving the energetics untouched or slight improved in strongly selfinteracting systems by the screened PZ correction; notably, for the KI class of functionals the potential energy surface is identical to the base functional, and thus such dependence is not relevant.

\section{CONCLUSIONS}

In conclusion we have shown how to extend the predictive power of Koopmans-compliant functionals to systems characterized by a complex electronic manifold, such as transition-metal complexes, finding that an orbital-dependent treatment of the screening is crucial to the correct description of spectral properties when orbitals with very different chemical character are considered. A scheme based on a linear-response approach has been introduced and discussed, highlighting the physical content behind the screening coefficient in Koopmanscompliant functionals. We found excellent agreement for the computed value of the ionization potentials with both experiment and state-of-the-art many-body perturbation theory, especially when the orbital dependence of the screening is correctly accounted for and when a localized representation of the electronic manifold is used to enforce the generalized PWL condition of KC functionals. The results on a carefully tested set of 41 transitionmetal complexes show mean absolute errors of $0.20,0.22$ and $0.29 \mathrm{eV}$, respectively, for the KI functional calculated on different localized representations; ten different many-body perturbation theory approaches were in the range $0.21 \mathrm{eV}$ to $1.92 \mathrm{eV}$, with $\mathrm{G}_{0} \mathrm{~W}_{0} @ \mathrm{PBE} 0$ being the most accurate one. These results reiterate the role of Koopmans' compliant functional as spectral functionals able to simultaneously reproduce electronic spectra and total energies, and as quasiparticle approximations to the exact spectral potentia 29230 .

\section{ACKNOWLEDGMENTS}

We acknowledge partial support from the Swiss National Centre for Computational Design and Discovery of Novel Materials (MARVEL). NC acknowledges Dr. Matteo Coccoccioni for useful discussions. We would like to thank Dr. Sabine Körbel for providing the equilibrium geometries.
${ }^{1}$ G. Onida, L. Reining, and A. Rubio, Reviews of Modern Physics 74, 601 (2002).

${ }^{2}$ J. P. Perdew, R. G. Parr, M. Levy, and J. L. Balduz, Physical Review Letters 49, 1691 (1982).
3 J. P. Perdew and M. Levy, Physical Review Letters 51, 1884 (1983).

${ }^{4}$ C.-O. Almbladh and U. von Barth, Physical Review B 31, 3231 (1985). 
5 J. P. Perdew and M. Levy, Physical Review B 56, 16021 (1997)

${ }^{\circ}$ R. W. Godby and I. D. White, Physical Review Letters 80, 3161 (1998)

7 J. P. Perdew, W. Yang, K. Burke, Z. Yang, E. K. U. Gross, M. Scheffler, G. E. Scuseria, T. M. Henderson, I. Y. Zhang, A. Ruzsinszky, H. Peng, J. Sun, E. Trushin, and A. Görling, PNAS 114, 2801 (2017)

8 M. Cococcioni and S. de Gironcoli, Physical Review B 71, 035105 (2005)

${ }^{9}$ A. J. Cohen, P. Mori-Sánchez, and W. Yang, Science 321, $792(2008)$

${ }^{10}$ H. J. Kulik, M. Cococcioni, D. A. Scherlis, and N. Marzari, Physical Review Letters 97, 103001 (2006).

11 P. Mori-Sánchez, A. J. Cohen, and W. Yang, The Journal of Chemical Physics 125, 201102 (2006).

${ }^{12}$ P. Mori-Sánchez, A. J. Cohen, and W. Yang, Physical Review Letters 100, 146401 (2008)

13 J. P. Perdew and A. Zunger, Physical Review B 23, 5048 (1981)

${ }^{14}$ X. Zheng, A. J. Cohen, P. Mori-Sánchez, X. Hu, and W. Yang, Physical Review Letters 107, 026403 (2011)

15 E. Kraisler and L. Kronik, Physical Review Letters 110, 126403 (2013).

10 A. Görling, Physical Review B 91, 245120 (2015)

17 C. Li, X. Zheng, A. J. Cohen, P. Mori-Sánchez, and W. Yang, Physical Review Letters 114, 053001 (2015)

18 T. Stein, H. Eisenberg, L. Kronik, and R. Baer, Physical Review Letters 105, 266802 (2010)

${ }^{19}$ L. Kronik, T. Stein, S. Refaely-Abramson, and R. Baer, Journal of Chemical Theory and Computation 8, 1515 (2012)

20 S. Refaely-Abramson, S. Sharifzadeh, N. Govind, J. Autschbach, J. B. Neaton, R. Baer, and L. Kronik, Physical Review Lettersrs 109, 226405 (2012).

${ }^{21}$ T. Shimazaki and Y. Asai, Chemical Physics Lettersrs 466, 91 (2008).

${ }_{22}$ J. H. Skone, M. Govoni, and G. Galli, Physical Review B 89, $195112(2014)$

23 N. P. Brawand, M. Vörös, M. Govoni, and G. Galli, Physical Review X 6, 041002 (2016)

${ }_{24}$ I. Dabo, M. Cococcioni, and N. Marzari, arXiv:0901.2637 [cond-mat] (2009), arXiv: 0901.2637.

25 I. Dabo, A. Ferretti, N. Poilvert, Y. Li, N. Marzari, and M. Cococcioni, Physical Review B 82, 115121 (2010).

${ }^{26}$ I. Dabo, A. Ferretti, G. Borghi, N. L. Nguyen, C.-H. Park, M. Cococcioni, and N. Marzari, Psi-K Newsletter 119 (2013)

27 I. Dabo, A. Ferretti, C.-H. Park, N. Poilvert, Y. Li, M. Cococcioni, and N. Marzari, Phys. Chem. Chem. Phys. 15, 685 (2012).

${ }_{28}$ G. Borghi, A. Ferretti, N. L. Nguyen, I. Dabo, and N. Marzari, Physical Review B 90, 075135 (2014)

29 A. Ferretti, I. Dabo, M. Cococcioni, and N. Marzari, Physical Review B 89, 195134 (2014).

so M. Gatti, V. Olevano, L. Reining, and I. V. Tokatly, Physical Review Letters 99, 057401 (2007)

${ }_{31}$ R. A. Heaton, J. G. Harrison, and C. C. Lin, Physical Review B 28, 5992 (1983).

${ }^{32}$ M. R. Pederson, R. A. Heaton, and C. C. Lin, The Journal of Chemical Physics 80, 1972 (1984)

33 M. R. Pederson, R. A. Heaton, and C. C. Lin, The Journal of Chemical Physics 82, 2688 (1985).
${ }^{34}$ M. R. Pederson and C. C. Lin, The Journal of Chemical Physics 88, 1807 (1988).

35 S. Lehtola and H. Jónsson, J. Chem. Theory Comput. 10, 5324 (2014)

36 S. F. Boys, Reviews of Modern Physics 32, 296 (1960)

37 N. Marzari, A. A. Mostofi, J. R. Yates, I. Souza, and D. Vanderbilt, Reviews of Modern Physics 84, 1419 (2012).

38 S. Goedecker and C. J. Umrigar, Physical Review A 55, 1765 (1997).

$3 y$ M. Stengel and N. A. Spaldin, Physical Review B 77, $155106(2008)$.

${ }^{40}$ G. Borghi, C.-H. Park, N. L. Nguyen, A. Ferretti, and N. Marzari, Physical Review B 91, 155112 (2015).

41 T. Körzdörfer, S. Kümmel, and M. Mundt, The Journal of Chemical Physics 129, 014110 (2008)

42 O. A. Vydrov, G. E. Scuseria, and J. P. Perdew, The Journal of Chemical Physics 126, 154109 (2007)

${ }^{43}$ O. A. Vydrov and G. E. Scuseria, The Journal of Chemical Physics 122, 184107 (2005)

${ }^{44}$ N. L. Nguyen, G. Borghi, A. Ferretti, I. Dabo, and N. Marzari, Physical Review Letters 114, 166405 (2015).

45 N. L. Nguyen, G. Borghi, A. Ferretti, and N. Marzari, J. Chem. Theory Comput. 12, 3948 (2016)

46 N. L. Nguyen, N. Colonna, A. Ferretti, and N. Marzari, arXiv:1708.08518 [cond-mat] (2017), arXiv: 1708.08518.

47 J. Ma and L.-W. Wang, Scientific Reports 6, 24924 (2016).

48 R. M. Martin, L. Reining, and D. M. Ceperley, Interacting Electrons (Cambridge University Press, 2016).

49 E. K. U. Gross and W. Kohn, Physical Review Letters 55, 2850 (1985).

${ }^{5}$ P. H. Dederichs, S. Blügel, R. Zeller, and H. Akai, Physical Review Letters 53, 2512 (1984)

${ }^{51}$ V. I. Anisimov and O. Gunnarsson, Physical Review B 43, 7570 (1991).

52 M. S. Hybertsen, M. Schlüter, and N. E. Christensen, Physical Review B 39, 9028 (1989)

53 A. K. McMahan, R. M. Martin, and S. Satpathy, Physical Review B 38, 6650 (1988)

54 J. F. Janak, Physical Review B 18, 7165 (1978)

55 This restriction can be however released (see Supporting Information) and the final result of the derivation [Eq. [8] apply to any equivalent representation of the KS orbitals, i.e. to any set of orbitals related to the KS one by a unitary transformation.

56 H. Hellmann, Einführung in die Quantenchemie (J.W. Edwards, Ann Arbor, Mich, 1944) open Library ID: OL21481721M.

57 R. P. Feynman, Physical Review 56, 340 (1939)

${ }^{58}$ M. Petersilka, U. J. Gossmann, and E. K. U. Gross, Physical Review Letters 76, 1212 (1996)

${ }^{59}$ R. F. Nalewajski, Density Functional Theory II: Relativistic and Time Dependent Extensions (Springer Verlag, 1996).

${ }^{60}$ V. I. Anisimov, J. Zaanen, and O. K. Andersen, Physical Review B 44, 943 (1991)

${ }^{\circ 1}$ V. I. Anisimov, I. V. Solovyev, M. A. Korotin, M. T. Czyżyk, and G. A. Sawatzky, Physical Review B 48, 16929 (1993)

${ }^{02}$ A. I. Liechtenstein, V. I. Anisimov, and J. Zaanen, Physical Review B 52, R5467 (1995).

${ }^{63}$ O. Gunnarsson, O. K. Andersen, O. Jepsen, and J. Zaanen, Physical Review B 39, 1708 (1989).

64 O. Gunnarsson, Physical Review B 41, 514 (1990) 
${ }^{65}$ M. Springer and F. Aryasetiawan, Physical Review B 57, $4364(1998)$

T. Kotani, J. Phys.: Condens. Matter 12, 2413 (2000)

67 F. Aryasetiawan, M. Imada, A. Georges, G. Kotliar, S. Biermann, and A. I. Lichtenstein, Physical Review B 70, 195104 (2004)

${ }^{58}$ F. Aryasetiawan, K. Karlsson, O. Jepsen, and U. Schönberger, Physical Review B 74, 125106 (2006).

${ }^{69}$ I. V. Solovyev and M. Imada, Physical Review B 71, $045103(2005)$

${ }^{70}$ J. H. Skone, M. Govoni, and G. Galli, Physical Review B 93, 235106 (2016)

${ }^{7}$ N. P. Brawand, M. Govoni, M. Vörös, and G. Galli, J. Chem. Theory Comput. (2017), 10.1021/acs.jctc.7b00368

12 S. Körbel, P. Boulanger, I. Duchemin, X. Blase, M. A. L. Marques, and S. Botti, J. Chem. Theory Comput. 10, 3934 (2014)

73 S. Baroni, S. de Gironcoli, A. Dal Corso, and P. Giannozzi, Reviews of Modern Physics 73, 515 (2001)

74 P. Giannozzi, S. Baroni, N. Bonini, M. Calandra, R. Car, C. Cavazzoni, D. Ceresoli, G. L. Chiarotti, M. Cococcioni, I. Dabo, A. D. Corso, S. d. Gironcoli, S. Fabris, G. Fratesi, R. Gebauer, U. Gerstmann, C. Gougoussis, A. Kokalj, M. Lazzeri, L. Martin-Samos, N. Marzari, F. Mauri, R. Mazzarello, S. Paolini, A. Pasquarello, L. Paulatto, C. Sbraccia, S. Scandolo, G. Sclauzero, A. P. Seitsonen, A. Smogunov, P. Umari, and R. M. Wentzcovitch, Journal of Physics: Condensed Matter 21, 395502 (2009)

${ }^{75}$ P. Giannozzi, O. Andreussi, T. Brumme, O. Bunau, M. B. Nardelli, M. Calandra, R. Car, C. Cavazzoni, D Ceresoli, M. Cococcioni, N. Colonna, I. Carnimeo, A. D. Corso, S. d. Gironcoli, P. Delugas, R. A. D. Jr, A Ferretti, A. Floris, G. Fratesi, G. Fugallo, R. Gebauer, U. Gerstmann, F. Giustino, T. Gorni, J. Jia, M. Kawamura, H-
Y Ko, A. Kokalj, E. Küçükbenli, M. Lazzeri, M. Marsili, N. Marzari, F. Mauri, N. L. Nguyen, H.-V. Nguyen, A Otero-de-la-Roza, L. Paulatto, S. Poncé, D. Rocca, R. Sabatini, B. Santra, M. Schlipf, A. P. Seitsonen, A. Smogunov, I Timrov, T. Thonhauser, P. Umari, N. Vast, $\mathrm{X}$. Wu, and S. Baroni, Journal of Physics: Condensed Matter 29, 465901 (2017).

76 D. R. Hamann, Physical Review B 88, 085117 (2013).

77 M. Schlipf and F. Gygi, Computer Physics Communications 196, 36 (2015)

78 "SG15 ONCV Potentials," (2017).

79 The equilibrium geometries of the transition metal complexes were kindly provided by the authors of Ref. 72 .

80 Y. Li and I. Dabo, Physical Review B 84, 155127 (2011)

81 J. P. Perdew, K. Burke, and M. Ernzerhof, Physical Review Letters 77, 3865 (1996)

82 For those experiments for which is unclear whether they are vertical or adiabatic (see Supporting Information for the complete list) the calculated value are left out of the average. In the case of adiabatic experimental value we used the same correction used in Ref. 72$]$ based on the difference between adiabatic and vertical $\triangle \mathrm{SCF}$ energies.

${ }^{83}$ X. Wu, H. Xie, Z. Qin, K. Tan, Z. Tang, and X. Lu, J. Phys. Chem. A 115, 6321 (2011)

${ }^{84}$ P. J. Linstrom and W. G. Mallard, eds., NIST Chemistry WebBook, NIST Standard Reference Database Number 69 (National Institute of Standards and Technology, Gaithersburg MD, 20899, 2005).

85 S. Lehtola, M. Head-Gordon, and H. Jónsson, J. Chem. Theory Comput. 12, 3195 (2016).

${ }^{86}$ H. J. Kulik and N. Marzari, The Journal of Chemical Physics 135, 194105 (2011)

87 H. Hsu, K. Umemoto, M. Cococcioni, and R. Wentzcovitch, Physical Review B 79, 125124 (2009) 